\title{
An FDTD-Based Tool for Simulation of Nonlinear Interactions of Guided Waves
}

\author{
Alireza Marandi, Poman P.M. So, Thomas E. Darcie \\ ECE Department, University of Victoria, Victoria, BC, Canada
}

\begin{abstract}
A new tool is proposed for simulation of spatial second-order nonlinear interactions of guided waves based on modified one-dimensional finite-difference time-domain (1-D FDTD) calculations with an embedded nonlinear susceptibility tensor. Parallel 1-D engines are used to calculate threedimensional behaviors according to propagation properties of the waveguides. The method has been used successfully to simulate difference-frequency mixing in GaAs for terahertz generation, and could be applied to a wide range of problems that could not be solved easily by conventional methods.
\end{abstract}

Index Terms - Finite difference time domain, nonlinear optics, waveguides

\section{INTRODUCTION}

Nonlinear optics has a wide range of applications in different fields such as communications and integrated photonics. Many devices use the nonlinear interactions between guided waves to enable different applications such as electro-optic modulation, all-optical wavelength conversion and signal processing [1]. Although studied extensively for decades, the design of novel components in this area still suffers from lack of accurate, fast, and comprehensive simulation tools.

Finite-difference time-domain (FDTD) technique, as a general tool for simulation of Maxwell's equations, has attracted a great attention for decades due to the simplicity and acceptable accuracy offered by the presence of powerful computers [2]. The modification of this method to include nonlinear processes is challenging due to the computational overhead of switching between frequency and time domain implementation

Approximations of frequency domain properties in time domain and using convolution are of the main ideas that have been proposed for simulation of nonlinear interactions in FDTD. Simulation of second and third-order nonlinearities are added to FDTD using nonlinear the Schrodinger equation in [3]. Another implementation of nonlinear FDTD is achieved using z-transform [4], and more recently, two dimensional implementation of FDTD for second and third-order nonlinearities is implemented [5]. All these methods work with scalar nonlinear coefficients and are limited to $43 \mathrm{~m}$ symmetric crystals [6] due to the absence of full tensor implementation. Also, the convolution based implementation adds significant computational overhead.
In this paper, we propose a method based on simple modifications on 1-D FDTD for simulation of spatially dependent nonlinear interactions of guided modes. We show that, by using waveguide properties obtained by conventional simulation or analytical methods, we can exploit accuracy, and low computational expense of 1-D FDTD to calculate the three-dimensional nonlinear behaviors. The algorithm in this paper is presented for second order nonlinear processes such as difference and sum frequency generations, but could be modified to be used for higher-order effects. The proposed technique provides a powerful tool for dealing with frequency dependent properties of the waveguides and materials by calculating the multimode interactions in time-domain, which enables us to represent the properties associated with a valid frequency interval as a mode. We demonstrate this technique using terahertz generation by difference frequency mixing in GaAs and show good performance of the technique in comparison to analytical calculations.

\section{II.APPROACH}

Nonlinear interactions in electromagnetic waves can be explained by Maxwell's curl equations,

$$
\begin{gathered}
\frac{\partial \mathbf{B}}{\partial t}=-\nabla \times \mathbf{E} \\
\frac{\partial \mathbf{D}}{\partial t}=\nabla \times \mathbf{H} .
\end{gathered}
$$

Where $\mathbf{D}$ is the vector form of electric flux density

$$
\mathbf{D}=\varepsilon_{r} \varepsilon_{0} \mathbf{E}+\mathbf{P}^{(N L)},
$$

containing the contribution of linear interactions in material (first term), and nonlinear polarization interactions (second term). Consequently equation 2 can be written as:

$$
\varepsilon_{r} \varepsilon_{0} \frac{\partial \mathbf{E}}{\partial t}+\frac{\partial \mathbf{P}^{(N L)}}{\partial t}=\nabla \times \mathbf{H}
$$

In this paper, we are concentrating on $\chi^{(2)}$ processes in the material. However, the method could be used for simulation of higher-order interactions. Based on the second order nonlinear susceptibility we have [1]: 


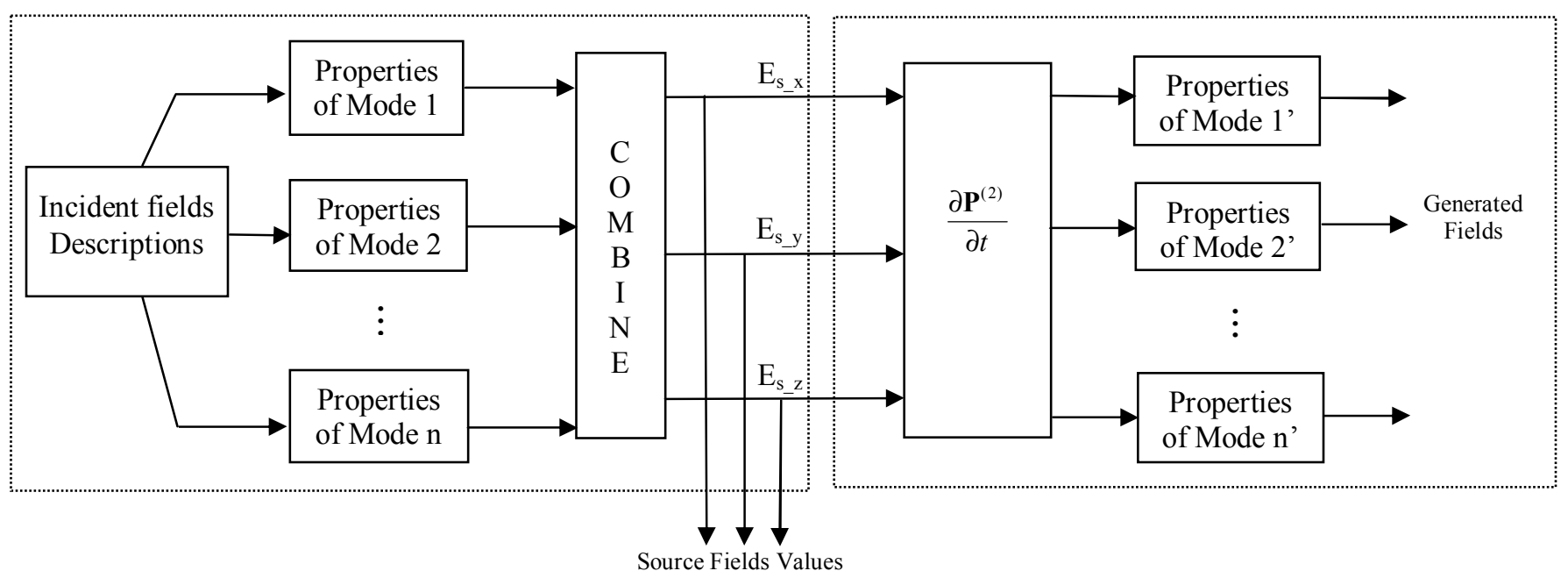

Fig. 1- The schematic diagram of the proposed nonlinear FDTD. The mode properties of the waveguide or waveguides involved in the process should be computed separately.

$$
\frac{\partial \mathbf{P}^{(2)}}{\partial t}=[\mathbf{T}] \times 2 \varepsilon_{0}\left[\begin{array}{llllll}
d_{11} & d_{12} & d_{13} & d_{14} & d_{15} & d_{16} \\
d_{21} & d_{22} & d_{23} & d_{24} & d_{25} & d_{26} \\
d_{31} & d_{32} & d_{33} & d_{34} & d_{35} & d_{36}
\end{array}\right] \times \frac{\partial}{\partial t}\left[\begin{array}{c}
E_{x^{\prime}}^{2} \\
E_{y^{\prime}}^{2} \\
E_{z^{\prime}}^{2^{\prime}} \\
E_{y^{\prime}} E_{z^{\prime}} \\
E_{x^{\prime}} E_{z^{\prime}} \\
E_{x^{\prime}} E_{y^{\prime}}
\end{array}\right] \text { (5) }
$$

where $\mathrm{E}_{\mathrm{x}}, \mathrm{E}_{\mathrm{y}}$, and $\mathrm{E}_{\mathrm{z}}$, are the components of electric field on the crystal axes and [T] is the matrix to transform the resulting polarization vector to the coordinate system used in equation 4 .

The proposed 1D-FDTD procedure for simulation of guided waves with nonlinear interactions is based on two assumptions. We first assume negligible depletion of incident fields. This is usually valid because the magnitudes of the generated fields are much smaller than the magnitudes of the incident fields, due to small nonlinear coefficients. The second assumption is orthogonal propagation properties of waveguide modes. Coupling between the supported modes is neglected, except the nonlinear coupling. This is valid for single-mode waveguides and most multimode waveguides.

According to the first assumption, the source fields and generated fields could be separated in the Maxwell's curl equations resulting in two set of new equations,

$$
\begin{gathered}
\frac{\partial \mathbf{B}_{s}}{\partial t}=-\nabla \times \mathbf{E}_{s} \\
\mathcal{E}_{r} \varepsilon_{0} \frac{\partial \mathbf{E}_{s}}{\partial t}=\nabla \times \mathbf{H}_{s}
\end{gathered}
$$

$$
\begin{gathered}
\frac{\partial \mathbf{B}_{g}}{\partial t}=-\nabla \times \mathbf{E}_{g} \\
\mathcal{E}_{r} \varepsilon_{0} \frac{\partial \mathbf{E}_{g}}{\partial t}+\frac{\partial \mathbf{P}^{(N L)}}{\partial t}=\nabla \times \mathbf{H}_{g}
\end{gathered}
$$

Where $\mathbf{E}_{\mathbf{s}}, \mathbf{B}_{\mathbf{s}}$, and $\mathbf{H}_{\mathbf{s}}$ refer to the incident field values, and $\mathbf{E}_{\mathrm{g}}, \mathbf{B}_{\mathrm{g}}$, and $\mathbf{H}_{\mathrm{g}}$ refer to the generated fields. According to the orthogonal mode assumption, the solution for the first set of equations, which doesn't contain a nonlinear process, could be approximated by plane wave propagation of waveguide modes. The properties of these guided modes could be obtained either analytically or by conventional simulation tools, and could be used for calculation of the source field values in the direction of propagation at each time step. However, the solution to the second set of equations, equations 8 , and 9 , for generated fields, is obtained using 1-D FDTD and considering the second term on the left hand side of equation 9 as a source term. This source term is calculated from the source field values given by equation 5 .

Figure 2 shows the schematic diagram of the proposed method. For the source fields calculation part, we know the waveguide properties for the incident fields. Each "mode properties" block refers to a unique set of mode properties valid for a specific part of the incident signal. For example, if the incident signal contains two optical wavelengths that propagate with the fundamental mode of the optical waveguide but with different effective indices (due to dispersion), for each wavelength a separate set of mode properties should be considered. The outputs of the "combine" block are the components of $\mathbf{E}_{\mathbf{s}}$ at each time step at all nodes in the direction of propagation. According to the computed source field values, the time derivative of nonlinear polarization is calculated by the backward difference method for all nodes, and it is fed to the 1-D FDTD calculations of all supported modes for the generated signals. 
For each mode properties block for the generated waves in the waveguide, we consider a modified 1D-FDTD formulation. For example for the y-polarized field propagating in the $\mathrm{x}$ direction, the equations for updating the field values would be as following:

$$
\begin{gathered}
\mu \frac{\left.H_{z}\right|_{x} ^{t+\Delta t}-\left.H_{z}\right|_{x} ^{t}}{\Delta t}=-\frac{\left.E_{y}\right|_{x+\frac{\Delta x}{2}} ^{t+\frac{\Delta t}{2}}-\left.E_{y}\right|_{x-\frac{\Delta x}{2}} ^{t+\frac{\Delta t}{2}}}{\Delta x} \\
\varepsilon_{r} \varepsilon_{0} \frac{\left.E_{y}\right|_{\mid t+\frac{\Delta x}{2}}+\left.E_{y}\right|_{x+\frac{\Delta t}{2}} ^{t+\frac{\Delta t}{2}}}{\Delta t}+\frac{\partial \mathbf{P}^{(N L)}}{\partial t}=\frac{\left.H_{z}\right|_{x+\Delta x} ^{t}-\left.H_{z}\right|_{x} ^{t}}{\Delta x}
\end{gathered}
$$

Where $\Delta \mathrm{t}$ is the time step and $\Delta \mathrm{x}$ is the mesh size. Electric field values, $E_{y}$, are calculated in positions starting from $\Delta x / 2$ and at time steps starting from $\Delta t / 2$. However, the magnetic field values are calculated at time steps and mesh steps starting from zero, to allow the central difference approximation for both time and space derivatives [2]. $\varepsilon_{\mathrm{r}}$ should be set as the effective dielectric constant of the corresponding mode. Loss of the modes also could be added to the equations when interpreted into effective electric or magnetic conductivities. The general equations for FDTD could be found in [2].

\section{RESULTS}

To verify the ability of the proposed tool in simulation of second-order nonlinear interaction of guided waves, it has been used to simulate terahertz generation by difference frequency mixing in GaAs. Phase matching for this process could be provided using integration of appropriate optical and terahertz waveguides by exploiting the dispersion properties of GaAs. In this example, perfect overlap between the modes is assumed to simply acquire comparable results to theoretical calculations. Theoretical calculations are based on the plane wave approximation using the equations provided in [1].

Two z-polarized optical wavelengths with the difference frequency of $2 \mathrm{THz}$ and central wavelength of $1.55 \mu \mathrm{m}$ have been applied to the waveguide. Considering the incident electric fields are parallel to $<111>$ direction of the crystal, the generated terahertz field values for all data points at a certain time step and for two cases of phase-matched and non-phasematched conditions are depicted in Figure 2. The amplitude of the incident fields are $2.3 \times 10^{6} \mathrm{~V} / \mathrm{m}$, the group index for optical wavelengths is 3.4 , and the effective index of the terahertz wave is 3.4 and 3.1 for phase-matched and nonphase-matched situations, respectively. The nonlinear FDTD method clearly reproduces the well known oscillatory behavior of difference frequency generation for non-phasematched condition, and the linear (in $\mathrm{x}$ ) growth for phasematched.

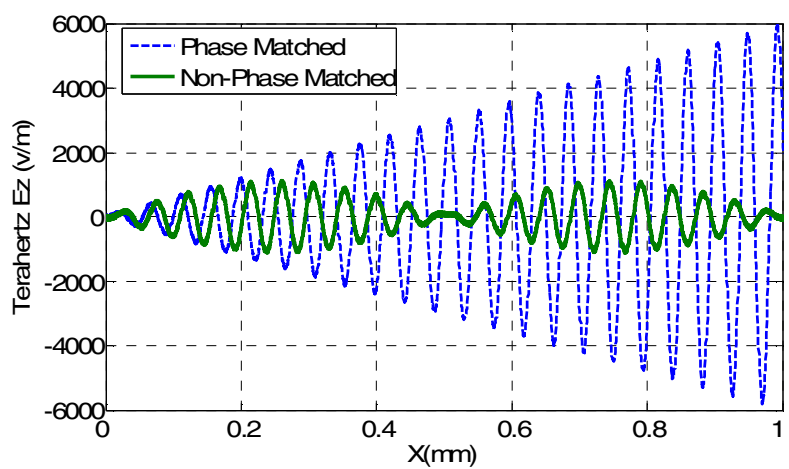

Fig. 2- The generated terahertz signal at a certain time step for two cases of phase-matched and non-phase-matched environment. (mesh size is $5 \times 10^{-7} \mathrm{~m}$ )

Figure 3 shows the comparison between the results obtained from the nonlinear FDTD method and theoretical calculation for terahertz generation in GaAs for perfect phase matching. The upper curves are for the crystal cut where the incident electric fields are parallel to the $<111>$ direction, and the lower curves are for fields parallel to the $<110>$ direction. The dependence on crystal orientation arises from transformation matrix $\mathbf{T}$ in equation 5, for nonlinear FDTD, and a different effective nonlinear coefficient for theoretical calculations.

The 1D mesh size for FDTD is $5 \times 10^{-7} \mathrm{~m}$. The resultea obtained for the lengths containing few mesh cells are far from the expected values. However, after passing about 200 mesh cells $\left(10^{-4} \mathrm{~m}\right)$ the difference become less than $2 \%$. The logarithmic scale is used for both axes to magnify the error for short distances and small values of electric field. The resulting oscillations in amplitude and the initial error are partially due to the sudden excitation of the structure, and could be compensated when the incident fields are smoothly applied in time. Figure 4 shows the resulting electric field value in the $y$ direction calculated in the $<111>$ case, showing calculation error of about $10^{-11} \mathrm{~V} / \mathrm{m}$.

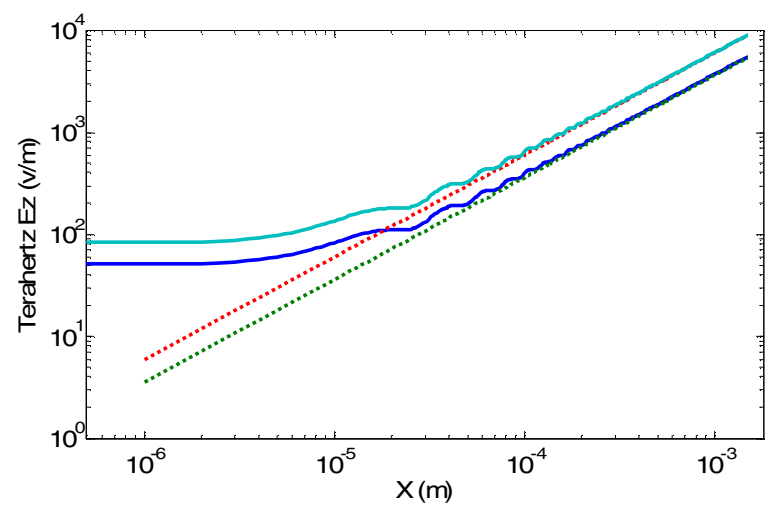

Fig. 3- Generated terahertz electric field magnitude calculated by nonlinear FDTD (solid line) and theoretical calculations (dash line). The upper curve is for crystal cut in $<111>$ direction and the lower curve is for $<110>$ direction. 


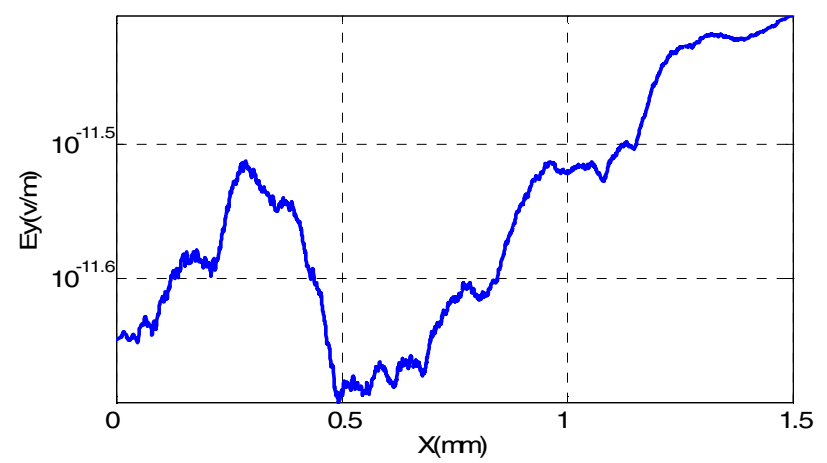

Fig. 4- The calculated Ey field by nonlinear FDTD, which is supposed to be zero theoretically.

\section{DISCUSSION}

The proposed method is based on a plane-wave approximation of the waveguide modes and electric field components of the modes which are perpendicular to the direction of waveguide. For TE and TEM modes, where the electric field is perpendicular to the direction of propagation, this approximation works well. However, for TM modes, we have two components of electric fields, one is perpendicular to and the other is in the direction of propagation. For lowerorder modes, the component in the direction of propagation is much smaller than the transverse component and can be neglected with acceptable accuracy. To increase accuracy for calculation of the nonlinear polarizations involving TM source modes in equation 5, both components can be considered. For the generated TM modes, only the perpendicular component is calculated by FDTD and the other component is determined according to the ratio available in mode properties.

Conventional one-dimensional FDTD results in exact solution of Maxwell's equation [2], and it is always stable under the Courant criterion. In the nonlinear FDTD method, the stability depends on the added term of nonlinear polarization in equation 9. Since the nonlinear coefficients for materials are usually very small, this term is regularly several orders of magnitude smaller than the other terms. No stability problem has been observed in cases considered.

Several nonlinear phenomena in which new frequencies are generated can be simulated with the proposed method, such as parametric amplification and oscillation, all-optical wavelength conversion, and electro-optic modulation. For other types of processes, such as soliton propagation, the algorithm should be modified to consider the effect of nonlinear processes on the incident signal (e.g. self-phase modulation), in addition to the generated signals. Overlap between the modes is an important issue that can be added to the method as appropriate coefficients to increase the accuracy.
A new FDTD-based simulation tool is proposed to simulate three-dimensional nonlinear interactions of guided waves. The accuracy of the technique is verified in simulation of terahertz generation via difference frequency mixing. The proposed algorithm is simple, accurate and fast; hence it is an attractive method for the simulation and optimization of nonlinear waveguide components. Simulation of second and higher-order processes in grating-assisted waveguides, quasiphase matched materials, and crystals with highly anisotropic tensor properties are potential applications of this method. These nonlinear processes are not easily incorporated into other conventional FDTD tools.

\section{REFERENCES}

[1] R. W. Boyd, Nonlinear Optics, Academic Press, 2003.

[2] A. Taflove, S. C. Hagness, Computational Electrodynamics: The Finite-Difference Time-Domain Method, Artech House, Boston, 2005.

[3] R. M. Joseph, A. Taflove, "FDTD Maxwell's equations models for nonlinear electrodynamics and optics," IEEE Transactions on Antenna and Propagations, Volume 45, no. 3, March 1997 Page(s):364 - 374.

[4] D. M. Sullivan," Nonlinear FDTD formulations using Z transforms," IEEE Transactions on Microwave Theory and Techniques, Volume 43, no. 3, March 1995 Page(s):676 - 682.

[5] C. M. Reinke, A. Jafarpour, B. Momeni, M. Soltani, S. Khorasani, A. Adibi, X. Yong,R. K. Lee, "Nonlinear finite-difference time-domain method for the simulation of anisotropic, $\chi^{(2)}, \chi^{(3)}$ optical effects," IEEE Journal of Lightwave Technology, Volume 14, no. 4, April 2002 Page(s): 480 - 482

[6] S. Namba, "Electro-optical effect of zincblende," Journal of the Optical Society of America, vol. 51, no. 1, Jan. 1961, pp.76-79.

\section{CONCLUSION}

